\title{
STEM Tomography and Surface Plasmon Imaging of a Au-Pd Bi-metallic Nanorod with Exotic Morphology
}

\author{
Qingxiao Wang ${ }^{1}$, Yihan $\mathrm{Zhu}^{2}$, Jianfeng Huang ${ }^{2}$ and $\mathrm{Yu} \mathrm{Han}^{2}$
}

1. Imaging and Characterization Core Lab, King Abdullah University of Science and Technology,
Thuwal 23955-6900, Saudi Arabia
2. Advanced Membranes and Porous Materials Center, Physical Sciences and Engineering Division,
King Abdullah University of Science and Technology, Thuwal 23955-6900, Saudi Arabia

Utilizing surface enhanced Raman spectroscopy (SERS) to in situ monitor a catalytic reaction requires a bi-functional platform which is both catalytically and plasmonically active. In this study, we developed a novel bimetallic nanostructure, featuring $\mathrm{Au}-\mathrm{Pd}$ alloy horns selectively grown on the end of $\mathrm{Au}$ nanorods (AuNR@AuPd), and employed electron tomography and electron energy loss spectroscopy (EELS) to visualize its 3-D morphology as well as the distribution of its surface plasmon. The results well explain the observed high catalytic activity and SERS sensitivity of this new material [1].

STEM tomography was carried out on a FEI Titan Super Twin electron microscope operated at $300 \mathrm{kV}$. A series of HAADF STEM images was acquired by tilting the specimen from $-75^{\circ}$ to $+75^{\circ}$ with $1^{\circ}$ intervals. The obtained image series was first aligned and then reconstructed by SIRT method in FEI Inspect3D software. The 3D volume rendering and isosurface construction of the reconstructed volume was performed in Avizo software. Figure. 1(a)-(c) shows the surface rendering of the AuNR@AuPd projected along [100], [110] and [001] respectively. It can be seen clearly from the tomography result that along the $[00 \pm 1]$ zone axis, the rod end terminated in a flattened pyramid. In Figure. 1(d), Detailed analysis from the high resolution HAADF STEM image shows that this flattened pyramid is enclosed by the $\left\{\begin{array}{lll}0 & 5 & 12\end{array}\right\}$ facet which agrees with a recent study [2].

STEM EELS Spectrum Imaging (SI) which can offer both good spatial resolution and energy resolution is an excellent technique for the surface plasmon analysis of materials at nanometer range. The EELS SI mapping of the AuNR@AuPd has been performed on FEI Titan Cubed electron microscope equipped with a monochromator, a CEOS probe-corrector and a Gatan Image Filter (GIF Tridiem). The energy resolution of the monochromated electron probe is $0.17 \mathrm{eV}$ as measured from the full-width at half-maximum (FWHM) of the zero loss peak (ZLP). An SI of 150 pixels $\times 60$ pixels size was acquired to generate a 3-D dataset. The exposure time for each pixel is $0.2 \mathrm{~s}$ and total acquisition time is about 30 min. The spatial drift has been corrected every $30 \mathrm{~s}$. Because of the instability of the microscope, ZLP of the spectra will shift a little bit during the SI acquisition and this can be corrected by applying a Gaussian peak fit at each pixel of the SI, then shifting spectral data accordingly.

Figure 2 (a) shows the EELS spectra at the end of rods and at the middle of the rods along [100] orientation. The peak maximum was found at $1.5 \mathrm{eV}$ for the rod ends, and at $2.2 \mathrm{eV}$ for the middle of rods. Figure 2 (c) shows a consistent result but from [110] orientation. 
Figure 2 (b) and (d) shows the 2D surface plasmon mapping of the AuNR@AuPd along [100] and [110] axis, respectively. The energy position of the mapping is at $1.5 \mathrm{eV}$ and $2.2 \mathrm{eV}$ with a $0.2 \mathrm{eV}$ energy window. It is clear to see from the result that the $1.5 \mathrm{eV}$ is highly localized at the two rod ends while the weakly excitations at $2.2 \mathrm{eV}$ was uniformly distributed near the rod body.

With the help of STEM tomography and EELS surface plasmon mapping, we confirm that the novel AuNR@AuPd nanostructure shows exotic crystal morphologies with high index facets and superior catalytic activity. And it can serve as an ideal bifunctional platform to in-situ monitor catalytic reactions with SERS.

\section{References:}

[1] Jianfeng Huang, Yihan Zhu et al, J. Am. Chem. Soc., 135, (2013), 8552-8561.

[2] Katz-Boon et al, Nano Lett., 11, (2011), 273-278.

[3] We acknowledge financial supports by research funds to Y.H. from King Abdullah University of Science and Technology.
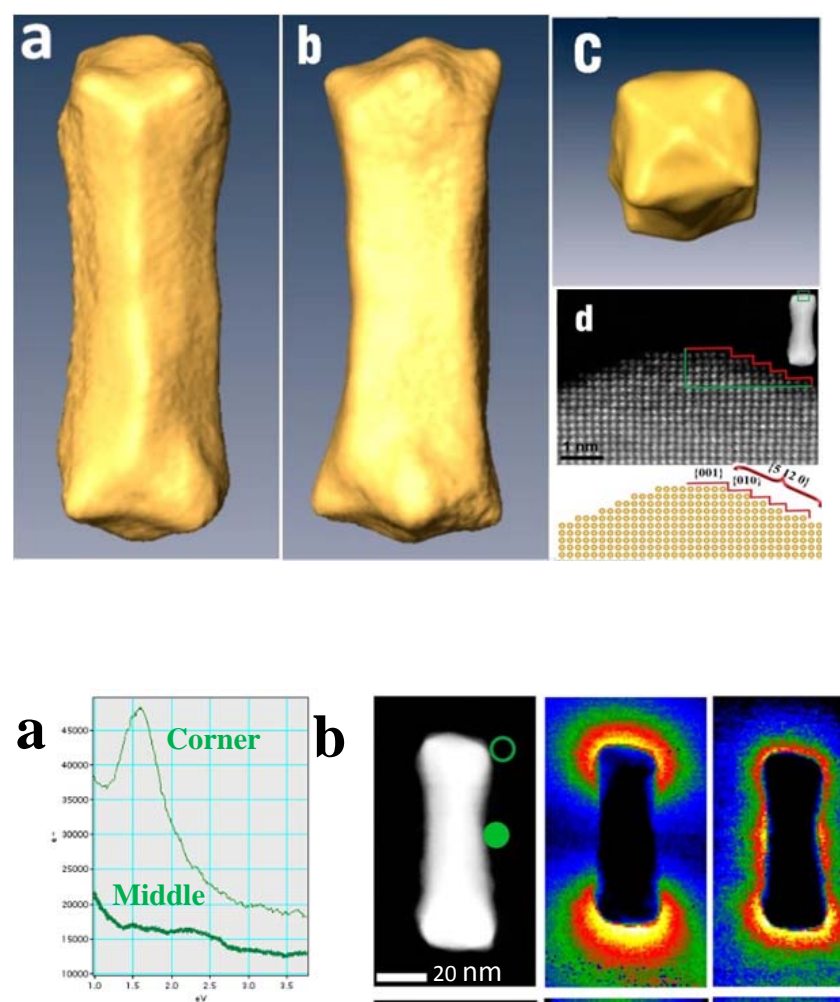

C
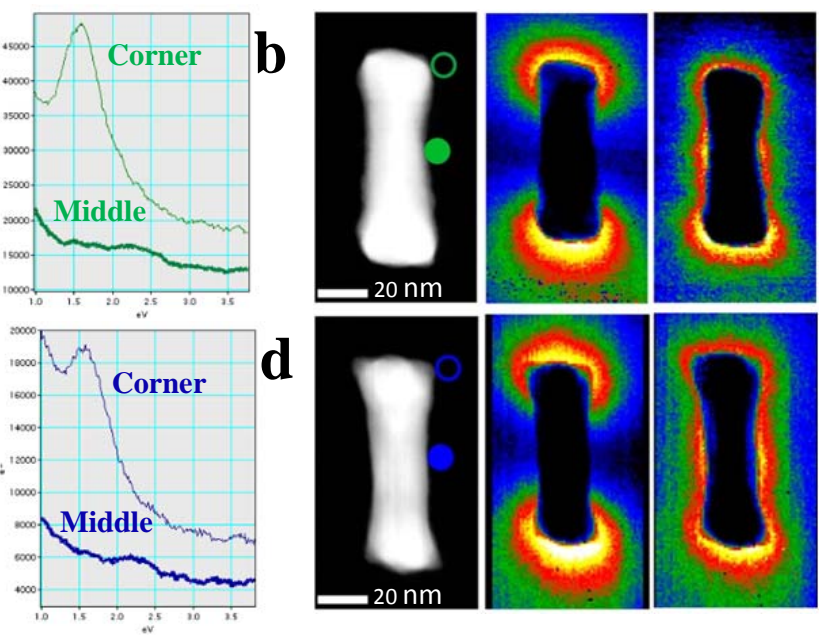

Figure. 1. Surface rendering of the AuNR@AuPd nanostructure reconstructed from HAADF-STEM tomography, viewed along (a) the [100], (b) the [110] and (c) the [001] zone axis. (d) STEM image of the flattened pyramid at the end of a rod. The calculated result shows that the pyramid has $\{0$ $512\}$ side facets.

Figure. 2. (a) EELS spectra of AuNR@AuPd at different positions labeled in the STEM images along [100] orientation. (b) STEM image (left) along [100] axis, surface plasmon mapping at $1.5 \mathrm{eV}$ (middle) and 2.2 $\mathrm{eV}$ (right). The energy window is $0.2 \mathrm{eV}$. (c) EELS spectra of AuNR@AuPd of different positions along [110] orientation. (d) STEM image (left) along [110] axis, surface plasmon mapping at $1.5 \mathrm{eV}$ (middle) and 2.2 $\mathrm{eV}$ (right). The energy window is $0.2 \mathrm{eV}$. 